\title{
Aquaporin-5 attenuates gene regulation by Th2 cytokines via inhibiting STAT6 phosphorylation
}

\author{
Tatsuro Ogawa, Shun Komori, Ichiro Horie, Yoichiro Isohama
}

Lab. Appl. Pharmacol., Facul. of Pharm. Sci., Tokyo Univ. of Sci., Japan

\section{[Background]}

Aquaporins (AQPs) are small, integral membrane proteins that facilitate water transport across cell membranes in response to osmotic gradients. Thirteen mammalian AQPs (AQP0-AQP12) have been identified and localized to various epithelial, endothelial and other tissues. Recent studies have revealed that AQPs regulate not only water transport, but also some basic cellular functions, such as growth and migration. In our previous study, we have defined that AQP5, an isoform selectively expressed in exocrine glands and alveolar epithelial cells, potentiates TNF- $\alpha$-induced cytokine expression. Then, in the present study, we examined the effect of AQP5 on Th2 cytokine induced gene expression.

[Methods]

To examine the effect of AQP5 expression on the response to Th2 cytokines, NIH-3T3 cells were used. Cells were transfected with the plasmid containing AQP5 cDNA, and treated with IL-13 or IL-4 (20 ng/ml). Gene expression and STAT6 phosphorylation induced by Th2 cytokines were measured by western blotting and quantitative RT-PCR methods, respectively. Eotaxin-1 promoter $(-1363 /+55)$ activity was measured by luciferase assay.

[Results]

In control cells, IL-13 induced remarkable increase in eotaxin-1 and monocyte chemotactic protein-1 (MCP-1) mRNA expression. Transfection of AQP5 cDNA into cells, resulted in dose-dependent suppression in IL-13 induced these mRNAs expression. AQP5 expression also suppressed IL-4- induced gene expression. In consistent with mRNA level, AQP5 expression suppressed IL-13- induced eotaxin-1 promoter activity, suggesting that AQP5 expression downregulate transcription of eotaxin-1. Then, we checked phosphorylation of STAT6, a major signaling pathway of Th2 cytokine receptors. Interestingly, IL-13- induced phosphorylation of STAT6 in AQP5-expressing cells was considerably lower than that in control cells. In addition, cycloheximide, a protein synthesis inhibitor, canceled AQP5-induced suppression in Th2 responses. These results suggested that AQP5 suppress the signaling of Th2 cytokines via new proteins.

\section{[Conclusions]}

In this study, we have demonstrated that AQP5 repressed Th2 cytokine-induced chemokine expression followed by STAT6 phosphorylation. Although further studies are needed to reveal physiological and pathophysiological significance of this new function, the regulation of Th2 cytokine signaling by AQP5 may provide a new insight into the treatment of Th2 cytokine-related diseases, such as asthma and allergic diseases. 